\title{
Divergência genética em linhagens de melancia
}

\author{
Flávio de França Souza ${ }^{1}$; Manoel Abilio de Queiróz ${ }^{2}$;Rita de Cássia S. Dias ${ }^{3}$ \\ ${ }^{1}$ Embrapa Rondônia, C. Postal 406, 78.900-970 Porto Velho-RO; E-mail: flaviofs@ cpafro.embrapa.br; ${ }^{2}$ DTCS-UNEB, C. Postal 171,

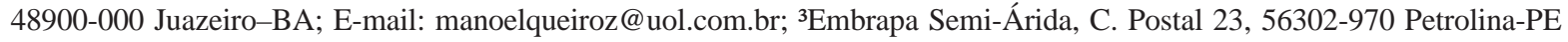

\section{RESUMO}

A divergência genética entre 31 genótipos de melancia foi avaliada por meio da análise de variáveis canônicas e de técnicas de agrupamento (Tocher e método hierárquico de Ward) baseadas na distância generalizada de Mahalanobis $\left(\mathrm{D}_{\mathrm{iii}}^{2}\right)$. Trinta linhagens, obtidas a partir de acessos coletados no Nordeste brasileiro e a cultivar 'Crimson Sweet' foram avaliadas quanto ao número de dias para o aparecimento da primeira flor masculina e da primeira flor feminina (NDM e NDF); número do nó da primeira flor masculina e da primeira flor feminina (NGM e NGF); número de frutos por planta (NFP); comprimento de rama principal (CRP); peso médio de fruto (PMF); teor de sólidos solúveis (TSS); diâmetro transversal e longitudinal do fruto (DTF e DLF) e espessura média de casca (EMC). O experimento foi realizado em delineamento de blocos ao acaso com três repetições, compostas por parcelas de sete plantas. As características que mais contribuíram para a divergência entre as linhagens foram número de frutos por planta, diâmetro longitudinal, teor de sólidos solúveis e peso médio de fruto. Foram formados três grupos por meio do método de otimização de Tocher, três por meio do método hierárquico de Ward e quatro grupos pela dispersão gráfica baseada nas duas primeiras variáveis canônicas. Neste caso, o grupo I compôs-se de sete linhagens de Pernambuco e uma da Bahia; o grupo II reuniu todas as 21 linhagens do Maranhão; os grupos III e IV foram compostos pela linhagem 97-0247.008 (Pernambuco) e pela cultivar Crimson Sweet, respectivamente. As linhagens 87019.021 e 87-019.022 foram as mais semelhantes, enquanto a linhagem 87-019.023 e 'Crimson Sweet' apresentaram maior dissimilaridade pela distância generalizada Mahalanobis $\left(\mathrm{D}^{2}{ }_{\mathrm{ii}}\right)$. Os cruzamentos mais promissores serão aqueles realizados entre Crimson Sweet e as linhagens do grupo II. Cruzamentos entre Crimson Sweet e as linhagens do grupo I serão interessantes para a obtenção de populações de plantas prolíficas e de frutos pequenos.

Palavras-chave: Citrullus lanatus, análise multivariada, análise de agrupamento, variáveis canônicas.

\section{ABSTRACT}

\section{Genetic divergence in watermelon lines}

Genetic dissimilarity among 31 watermelon genotypes was evaluated through the canonic analysis and cluster analysis (Tocher Method and Ward Method) based on Mahalanobis distance $\left(\mathrm{D}_{\mathrm{ii}}^{2}\right)$ Thirty lines obtained from accesses collected in the Northeast of Brazil and the cultivar Crimson Sweet were evaluated to determine the number of days to appearance of the first male and female flower (NDM and NDF); number of shoots to the appearance of the first male and female flower (NGM and NGF); number of fruits per plant (NFP); length of the main vine (CRP); fruit mean weight (PMF); total solid soluble content (TSS); transversal and axial fruit diameter (DTF and DLF) and rind thickness average (EMC). The experiment was carried out in a randomized block design with three replicates and seven plants per plot. The characteristics that contributed more to the genetic dissimilarity among the genotypes were number of fruit per plant, axial diameter of fruit, total solid soluble content and fruit mean weight. Three clusters were formed by Tocher's optimization method and by the Ward Method, while four clusters were formed by the graphic dispersion, based on two first canonic variables. In the last case, cluster I was composed of seven lines from Pernambuco and one line from Bahia; cluster II was formed by all the 21 lines from Maranhão; clusters III and IV were composed of line 97-0247.008 (Pernambuco) and of 'Crimson Sweet', respectively. Lines 87-019.021 and 87-019.022 were the closest related, while line 87-019.023 and Crimson Sweet presented the biggest dissimilarity by the Mahalanobis distance $\left(\mathrm{D}_{\mathrm{ii}}^{2}\right)$. The hybridization among 'Crimson Sweet' and the lines from the cluster II will be the most promising. Hybridization among 'Crimson Sweet' and the lines from cluster I will be interesting to obtain populations of prolific plants, which give small fruits.

Keywords: Citrullus lanatus, multivariated analysis, cluster analysis, canonic analysis.

\section{(Recebido para publicação em 11 de setembro de 2003 e aceito em 29 de outubro de 2004)}

\begin{abstract}
A melancia [Citrullus lanatus (Thunb.) Matsum. \& Nakai] é uma cucurbitácea de grande importância econômica, sendo cultivada em vários países. Segundo dados da FAO (2002), em 2002, os maiores produtores foram China, Turquia, Irã, Estados Unidos e Egito. Entre os 99 países listados, o Brasil, ocupou a décima colocação, com produção de 620.000 toneladas e área colhida de aproximadamente $82.000 \mathrm{~h}$. Os principais estados produtores são BA, RS, SP, GO e PE (IBGE, 2003).
\end{abstract}

Apenas algumas poucas cultivares de melancia predominam em grande parte da área plantada. Além disso, a maioria destas cultivares provém de germoplasma bastante aparentado, agravando o estreitamento da base genética deste cultivo no país (QUEIROZ et al., 1999).

Considerando a variabilidade encontrada nos acessos coletados na região Nordeste, percebe-se que o uso do germoplasma local, como fonte de matéria prima para o melhoramento de melancia, surge como alternativa promissora, sobretudo para a obtenção de genótipos adaptados e resistentes aos principais estresses bióticos da cultura nas condições brasileiras (QUEIRÓZ, 1998).

Para realização de um programa eficiente de melhoramento é necessário reunir o maior número possível de informações relevantes sobre o germoplasma a ser utilizado. Portanto, a relação entre os progenitores envolvidos em cruzamentos será útil na identificação das combinações híbridas mais promissoras e na visualização do potencial genético de futuras linhagens (GERALDI; MIRANDA FILHO, 1988; CRUZ; VENCOVSKY, 1989). 


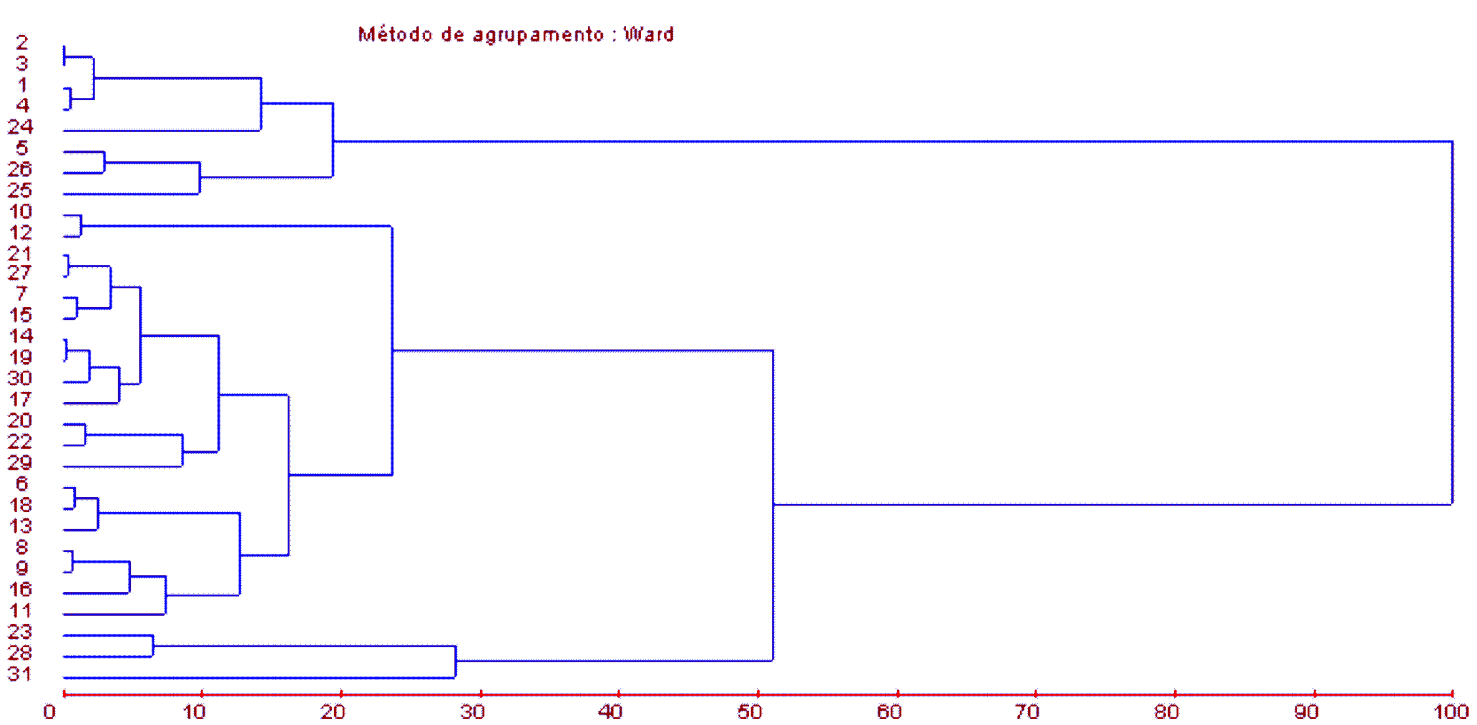

Legenda: $01=87-019.017 ; 02=87-019.021 ; 03=87-019.022 ; 04=87-019.023 ; 05=87-019.027 ; 06=91-109.059 ; 07=91-$ $109.060 ; 08=91-109.087 ; 09=91-109.118 ; 10=91-074.015 ; 11=91-074.029 ; 12=91-074.012 ; 13=91-079.001 ; 14=91-$ $079.008 ; 15=91-079.009 ; 16=91-079.010 ; 17=91-079.044 ; 18=91-079.045 ; 19=91-079.060 ; 20=91-099.011 ; 21=91-$ 099.023; $22=91-099.036 ; 23=97-0247.008 ; 24=97-0249.006 ; 25=97-0251.005 ; 26=97-0251.017 ; 27=97-0257.005 ; 28=97-$ 0265.021; $29=97-0263.029 ; 30=97-0207.002$ e $31=$ Crimson Sweet.

Figura 1. Representação gráfica da divergência genética entre 31 linhagens de melancia, obtida por meio do método hierárquico de Ward, com base na distância generalizada de Mahalanobis. Petrolina, Embrapa Semi-Árido, 1997.

A divergência genética em um grupo de progenitores tem sido avaliada com o objetivo de identificar as combinações híbridas de maior efeito heterótico e maior heterozigose, de modo que em suas populações segregantes sejam recuperados os genótipos superiores (CRUZ; REGAZZI, 1997). A avaliação da divergência genética tem sido realizada utilizando-se técnicas biométricas, processos preditivos e também por meio de marcadores bioquímicos e moleculares. Informações mais detalhadas sobre o emprego das técnicas bioquímicas e moleculares no estudo da divergência genética em melancia são apresentadas nos trabalhos de Assis (1994), Lee et al. (1996), Jarret et al. (1997) e Guerra-Sanz (2002).

Os métodos preditivos tomam por base diferenças fenotípicas apresentadas pelos progenitores, que podem ser quantificadas em termos de medidas de dissimilaridade. Dentre os mais utilizados pelos fitomelhoristas, estão a análise por variáveis canônicas e os métodos aglomerativos. A análise de variáveis canônicas é um processo de avaliação do grau de similaridade genética entre um grupo de progenitores que leva em consideração a covariância residual e a covariância fenotípica entre as características avaliadas (CRUZ; REGAZZI, 1997).
Os métodos aglomerativos têm como objetivo a reunião dos progenitores em grupos, de modo que haja homogeneidade dentro do grupo e heterogeneidade entre os grupos. Estes métodos dependem da estimativa prévia de medidas de dissimilaridade como a distância Euclidiana ou a distância generalizada de Mahalanobis $\left(\mathrm{D}_{\mathrm{ii}}^{2}\right)$, entre outras. Os métodos aglomerativos podem ser hierárquicos ou de otimização.

Nos métodos hierárquicos, o agrupamento dos progenitores é realizado por meio de um processo que se repete em vários níveis até que seja construído o dendrograma, que permitirá estabelecer a relação entre os progenitores. O método do vizinho mais próximo (Single Linkage Method), assim como o Método de Ward, são métodos hierárquicos nos quais os progenitores mais similares são identificados e agrupados por meio da matriz de dissimilaridade. À medida que os grupos vão sendo formados ou expandidos, a matriz vai sendo reduzida até que, finalmente, todos os progenitores são incluídos no mesmo grupo.

Entre os métodos de otimização, o método de Tocher é um dos mais utilizados pelos melhoristas. Nesse caso, assume-se que a média das medidas de dissimilaridade dentro do grupo é menor que as distâncias médias entre os grupos. Após a formação do grupo inicial, pela identificação do par de progenitores mais similar, é avaliada a possibilidade de inclusão dos outros progenitores, considerando o critério assumido anteriormente (CRUZ; REGAZZI, 1997).

O presente trabalho teve como objetivo a determinação da divergência genética entre linhagens de melancia, obtidas a partir de populações coletadas na região Nordeste do Brasil, por meio de técnicas de análises multivariadas.

\section{MATERIAL E MÉTODOS}

O experimento foi realizado na EE da Embrapa Semi-Árido, em Petrolina (PE), a uma latitude de $09^{\circ} 09^{\prime} \mathrm{S}$, longitude de $40^{\circ} 22^{\prime} \mathrm{W}$ e altitude de $360 \mathrm{~m}$. A região apresenta clima semi-árido, quente (BSh) e solo do tipo Latossolo Amarelo. Foram utilizadas 30 linhagens de melancia obtidas a partir de acessos coletados nos municípios de Pastos Bons, São João dos Patos e Paraibano, no Maranhão; Irecê, na Bahia e Petrolina em Pernambuco (Tabela 1). Também foi utilizada uma linhagem de autofecundação da cultivar Crimson Sweet. Empregou-se delineamento de blocos casualizados com três repeti- 
Tabela 1. Peso médio de fruto (PMF), teor de sólido solúveis (TSS), diâmetro transversal de fruto (DTF), diâmetro longitudinal (DLF), espessura média de casca (EMC), número de frutos por planta (NFP), número de dias para o aparecimento da primeira flor masculina (NDM); Número da gema que originou a primeira flor masculina (NGM), número de dias para o aparecimento da primeira flor feminina (NDF); número da gema que originou a primeira flor feminina (NGF) e comprimento da rama principal (CRP), de 30 linhagens e uma cultivar de melancia. Petrolina, Embrapa Semi-Árido, 1997.

\begin{tabular}{|c|c|c|c|c|c|c|c|c|c|c|c|c|}
\hline Linhagem & rigem $^{1}$ & $\begin{array}{c}\text { PMF }^{2} \\
\text { (kg) }\end{array}$ & $\begin{array}{c}\text { TSS } \\
\left({ }^{\circ} \text { brix }\right)\end{array}$ & $\begin{array}{l}\text { DTF } \\
(\mathrm{cm})\end{array}$ & $\begin{array}{l}\text { DLF } \\
(\mathrm{cm})\end{array}$ & $\begin{array}{l}\text { EMC } \\
(\mathrm{cm})\end{array}$ & $\begin{array}{c}\text { NFP } \\
\text { (unid) }\end{array}$ & $\begin{array}{l}\text { NDM } \\
\text { (unid) }\end{array}$ & $\begin{array}{l}\text { NGM } \\
\text { (unid) }\end{array}$ & $\begin{array}{l}\text { NDF } \\
\text { (unid) }\end{array}$ & $\begin{array}{c}\text { NGF } \\
\text { (unid) }\end{array}$ & $\begin{array}{l}\text { CRP } \\
\text { (m) }\end{array}$ \\
\hline$(87-019.017)$ & 1 & $2,16 \mathrm{e}$ & $4,9 d$ & $15,4 \mathrm{E}$ & $16,4 \mathrm{~d}$ & $0,97 \mathrm{~b}$ & $13,0 \mathrm{a}$ & 42,7 & $6,7 \mathrm{c}$ & $51,0 \mathrm{a}$ & $18,0 \mathrm{a}$ & $6,17 \mathrm{a}$ \\
\hline (87-019.021) & 1 & $2,27 \mathrm{e}$ & $5,0 \mathrm{~d}$ & $15,7 \mathrm{E}$ & $16,9 d$ & $1,03 \mathrm{~b}$ & $14,0 \mathrm{a}$ & 44,7 & $8,0 \mathrm{~b}$ & $53,0 \mathrm{a}$ & $17,7 \mathrm{a}$ & $5,09 \mathrm{~b}$ \\
\hline (87-019.022) & 1 & $1,90 \mathrm{e}$ & $5,0 d$ & $14,8 \mathrm{E}$ & $15,6 \mathrm{~d}$ & $0,90 \mathrm{~b}$ & $13,3 \mathrm{a}$ & 44,3 & $7,7 \mathrm{~b}$ & $53,0 \mathrm{a}$ & $17,3 \mathrm{a}$ & $4,96 \mathrm{~b}$ \\
\hline$(87-019.023)$ & 1 & $1,97 \mathrm{e}$ & $4,8 \mathrm{~d}$ & $14,7 \mathrm{E}$ & $15,9 \mathrm{~d}$ & $0,87 \mathrm{~b}$ & $14,8 \mathrm{a}$ & 43,7 & $7,3 \mathrm{~b}$ & $51,3 a$ & $16,3 \mathrm{a}$ & $6,21 \mathrm{a}$ \\
\hline (87-019.027) & 1 & $2,21 \mathrm{e}$ & $5,1 d$ & $15,6 \mathrm{E}$ & $16,7 d$ & $1,00 \mathrm{~b}$ & $11,4 \mathrm{~b}$ & 41,3 & $6,7 \mathrm{c}$ & $52,0 \mathrm{a}$ & $17,7 \mathrm{a}$ & $6,62 \mathrm{a}$ \\
\hline (91-109.059) & 2 & $5,33 \mathrm{c}$ & $7,3 \mathrm{~b}$ & $16,5 \mathrm{D}$ & $37,0 \mathrm{~b}$ & $1,43 a$ & $2,7 \mathrm{c}$ & 44,0 & $4,3 \mathrm{c}$ & $50,3 a$ & $15,0 \mathrm{~b}$ & $3,77 \mathrm{~b}$ \\
\hline$(91-109.060)$ & 2 & $5,91 \mathrm{c}$ & $7,2 \mathrm{~b}$ & $17,8 \mathrm{D}$ & $37,9 \mathrm{~b}$ & $1,60 \mathrm{a}$ & $3,1 \mathrm{c}$ & 42,0 & $5,7 \mathrm{c}$ & $47,7 \mathrm{~b}$ & 6,3 a & $5,05 \mathrm{~b}$ \\
\hline (91-109.087) & 2 & $5,36 \mathrm{c}$ & $7,0 \mathrm{~b}$ & $15,8 \mathrm{E}$ & $37,7 \mathrm{~b}$ & $1,33 \mathrm{a}$ & $3,4 \mathrm{c}$ & 41,3 & $5,0 \mathrm{c}$ & $47,0 \mathrm{~b}$ & $16,7 \mathrm{a}$ & $4,84 \mathrm{~b}$ \\
\hline$(91-109.118)$ & 2 & $6,38 \mathrm{~b}$ & $7,3 \mathrm{~b}$ & $16,0 \mathrm{E}$ & $38,8 \mathrm{~b}$ & $1,43 a$ & $2,9 \mathrm{c}$ & 39,3 & $4,3 \mathrm{c}$ & $46,3 \mathrm{~b}$ & $16,7 \mathrm{a}$ & $4,71 b$ \\
\hline (91-074.015) & 2 & $6,67 \mathrm{~b}$ & 7,2 b & $17,0 \mathrm{D}$ & $47,1 \mathrm{a}$ & $1,27 \mathrm{~b}$ & $4,5 \mathrm{c}$ & 42,0 & $7,0 \mathrm{~b}$ & $50,7 \mathrm{a}$ & $17,7 a$ & $4,97 \mathrm{~b}$ \\
\hline (91-074.029) & 2 & $6,99 \mathrm{~b}$ & $7,0 \mathrm{~b}$ & $18,3 \mathrm{D}$ & $39,2 \mathrm{~b}$ & $1,23 \mathrm{~b}$ & $3,5 \mathrm{c}$ & 38,7 & $6,0 \mathrm{c}$ & $46,3 \mathrm{~b}$ & $17,0 \mathrm{a}$ & 5,32 b \\
\hline (91-074.012) & 2 & $6,63 \mathrm{~b}$ & $7,1 \mathrm{~b}$ & $15,6 \mathrm{E}$ & $44,5 \mathrm{a}$ & $1,17 \mathrm{~b}$ & $3,5 \mathrm{c}$ & 41,0 & $6,3 \mathrm{c}$ & $48,3 \mathrm{~b}$ & $16,0 \mathrm{a}$ & $5,15 b$ \\
\hline (91-079.001) & 3 & $6,41 b$ & $8,2 \mathrm{~b}$ & $17,0 \mathrm{D}$ & $38,7 \mathrm{~b}$ & $1,87 \mathrm{a}$ & $2,7 \mathrm{c}$ & 48,3 & $5,3 c$ & $54,7 \mathrm{a}$ & $13,7 \mathrm{~b}$ & $3,73 \mathrm{~b}$ \\
\hline (91-079.008) & 3 & $5,24 \mathrm{c}$ & 7,7 b & $17,2 \mathrm{D}$ & $34,6 \mathrm{c}$ & $1,27 \mathrm{~b}$ & $3,0 \mathrm{c}$ & 41,3 & $4,7 \mathrm{c}$ & $48,0 \mathrm{~b}$ & $15,0 \mathrm{~b}$ & $4,76 \mathrm{~b}$ \\
\hline (91-079.009) & 3 & $6,54 \mathrm{~b}$ & $7,9 \mathrm{~b}$ & $18,3 \mathrm{D}$ & $36,9 \mathrm{~b}$ & $1,77 \mathrm{a}$ & $3,1 \mathrm{c}$ & 43,7 & $6,7 \mathrm{c}$ & $48,7 \mathrm{~b}$ & $15,3 \mathrm{~b}$ & $4,94 \mathrm{~b}$ \\
\hline (91-079.010) & 3 & $7,11 b$ & $7,9 \mathrm{~b}$ & $17,0 \mathrm{D}$ & $41,1 b$ & $1,87 \mathrm{a}$ & $3,0 \mathrm{c}$ & 39,0 & $4,3 \mathrm{c}$ & $45,0 \mathrm{~b}$ & $13,0 \mathrm{~b}$ & 5,32 b \\
\hline (91-079.044) & 3 & $5,73 \mathrm{c}$ & $7,6 \mathrm{~b}$ & $17,1 \mathrm{D}$ & $36,0 \mathrm{~b}$ & $1,47 \mathrm{a}$ & $3,7 \mathrm{c}$ & 50,7 & $8,0 \mathrm{~b}$ & 54,7 a & 16,3 a & $4,23 \mathrm{~b}$ \\
\hline (91-079.045) & 3 & $5,97 \mathrm{c}$ & $7,5 \mathrm{~b}$ & $16,8 \mathrm{D}$ & $38,2 \mathrm{~b}$ & $1,47 \mathrm{a}$ & $2,9 \mathrm{c}$ & 45,0 & $6,0 \mathrm{c}$ & $52,7 \mathrm{a}$ & $15,7 \mathrm{a}$ & $4,35 \mathrm{~b}$ \\
\hline (91-079.060) & 3 & $5,57 \mathrm{c}$ & $7,9 \mathrm{~b}$ & $17,5 \mathrm{D}$ & $35,7 \mathrm{~b}$ & $1,43 a$ & $3,9 \mathrm{c}$ & 43,0 & $6,0 \mathrm{c}$ & $48,7 \mathrm{~b}$ & $14,3 \mathrm{~b}$ & $4,55 \mathrm{~b}$ \\
\hline (91-099.011) & 4 & $3,99 \mathrm{~d}$ & $6,3 c$ & $15,7 \mathrm{E}$ & $27,5 \mathrm{c}$ & $1,37 \mathrm{a}$ & $2,5 \mathrm{c}$ & 45,0 & $6,3 \mathrm{c}$ & $51,7 \mathrm{a}$ & $13,3 \mathrm{~b}$ & $4,59 \mathrm{~b}$ \\
\hline (91-099.023) & 4 & $5,56 \mathrm{c}$ & $7,4 \mathrm{~b}$ & $17,3 \mathrm{D}$ & $31,8 \mathrm{c}$ & $1,47 \mathrm{a}$ & $2,8 \mathrm{c}$ & 41,0 & $4,7 \mathrm{c}$ & $46,7 \mathrm{~b}$ & $13,7 \mathrm{~b}$ & $5,03 \mathrm{~b}$ \\
\hline (91-099.036) & 4 & $5,00 \mathrm{c}$ & $6,5 \mathrm{c}$ & $17,1 \mathrm{D}$ & $31,7 \mathrm{c}$ & $1,50 \mathrm{a}$ & $2,6 \mathrm{c}$ & 42,0 & $5,7 \mathrm{c}$ & 51,3 a & $15,0 \mathrm{~b}$ & 5,97 a \\
\hline$(97-0247.008)$ & 1 & $9,31 a$ & $8,3 \mathrm{~b}$ & $22,4 \mathrm{~B}$ & $33,0 \mathrm{c}$ & $1,37 \mathrm{a}$ & $1,7 \mathrm{c}$ & 42,0 & $4,7 \mathrm{c}$ & $50,0 \mathrm{a}$ & $15,7 a$ & $5,84 a$ \\
\hline$(97-0249.006)$ & 5 & $1,93 \mathrm{e}$ & $6,1 c$ & $15,4 \mathrm{E}$ & $15,3 d$ & $1,13 b$ & $12,4 \mathrm{~b}$ & 44,7 & $11,0 \mathrm{a}$ & 56,3 a & 20,7 a & $7,18 \mathrm{a}$ \\
\hline$(97-0251.005)$ & 1 & $3,54 \mathrm{~d}$ & $6,6 \mathrm{c}$ & $17,4 \mathrm{D}$ & $21,8 d$ & $0,80 \mathrm{~b}$ & $7,1 \mathrm{c}$ & 43,3 & $5,7 \mathrm{c}$ & $49,7 \mathrm{a}$ & $16,0 \mathrm{a}$ & $5,76 \mathrm{a}$ \\
\hline$(97-0251.017)$ & 1 & $3,02 d$ & $6,6 \mathrm{c}$ & $16,9 \mathrm{D}$ & $20,5 d$ & $1,00 \mathrm{~b}$ & $11,3 \mathrm{~b}$ & 42,7 & $5,7 \mathrm{c}$ & 50,7 a & $17,0 \mathrm{a}$ & $6,27 \mathrm{a}$ \\
\hline$(97-0257.005)$ & 2 & $6,49 \mathrm{~b}$ & $7,2 \mathrm{~b}$ & $17,8 \mathrm{D}$ & $33,0 \mathrm{c}$ & $1,47 \mathrm{a}$ & $2,8 \mathrm{c}$ & 41,7 & $5,7 \mathrm{c}$ & $46,7 \mathrm{~b}$ & $14,3 \mathrm{~b}$ & $4,80 \mathrm{~b}$ \\
\hline$(97-0265.021)$ & 2 & $7,52 \mathrm{~b}$ & $8,0 \mathrm{~b}$ & $20,0 \mathrm{C}$ & $32,5 \mathrm{c}$ & $1,53 \mathrm{a}$ & $2,5 \mathrm{c}$ & 38,7 & $6,3 c$ & $46,3 \mathrm{~b}$ & $15,0 \mathrm{~b}$ & $5,70 \mathrm{a}$ \\
\hline$(97-0263.029)$ & 2 & $5,42 \mathrm{c}$ & $7,0 \mathrm{~b}$ & $17,3 \mathrm{D}$ & $39,5 \mathrm{~b}$ & $1,60 \mathrm{a}$ & $3,5 c$ & 40,7 & $5,0 \mathrm{c}$ & $43,3 \mathrm{~b}$ & $10,0 \mathrm{c}$ & $4,63 \mathrm{~b}$ \\
\hline$(97-0207.002)$ & 2 & $4,97 \mathrm{c}$ & $7,8 \mathrm{~b}$ & $16,8 \mathrm{D}$ & $37,0 \mathrm{~b}$ & $1,20 \mathrm{~b}$ & $2,5 \mathrm{c}$ & 43,0 & $6,3 \mathrm{c}$ & $50,3 a$ & $17,0 \mathrm{a}$ & $4,47 \mathrm{~b}$ \\
\hline Crimson Sweet & - & $10,31 a$ & $10,9 a$ & $25,5 \mathrm{~A}$ & $28,1 \mathrm{c}$ & $1,33 a$ & $1,3 \mathrm{c}$ & 39,3 & $5,0 \mathrm{c}$ & $43,7 \mathrm{~b}$ & $13,7 \mathrm{~b}$ & $4,76 \mathrm{~b}$ \\
\hline Média & & 5,27 & 7,0 & 17,2 & 31,5 & 1,33 & 5,3 & 42,6 & 6,1 & 49,5 & 15,7 & 5,15 \\
\hline C.V. (\%)3 & & 18,30 & 8,8 & 6,5 & 11,3 & 19,19 & 25,6 & 7,3 & 20,6 & 5,7 & 11,0 & 14,23 \\
\hline$\overline{\mathrm{F}^{4}}$ & & 14,50 ** & $12,3^{* *}$ & $11,1^{\text {** }}$ & 21,2 ** & 3,6 ** & $29,7^{* *}$ & $2,1 \mathrm{~ns}$ & $3,7 * *$ & 3,8 ** & $3,9 * *$ & $3,6^{* *}$ \\
\hline
\end{tabular}

${ }^{1 / 1} 1$ = Petrolina (PE); 2 = Pastos Bons (MA); 3 = Paraibano (MA); 4 = São João dos Patos (MA); 5 = Irecê (BA); ${ }^{2 / M e ́ d i a s ~ c o m ~ a ~ m e s m a ~ l e t r a, ~}$ na coluna, não diferem pelo teste de Scott \& Knott (1974) em nível de $5 \%$ de probabilidade; ${ }^{3 / C V}=$ Coeficiente de variação; ${ }^{4 / n s}=$ não significativo; $* *=$ significativo em nível de $1 \%$ de probabilidade

ções formadas por parcelas de sete plantas com espaçamento de 3,0 x 1,0 m.

Empregou-se sistema de irrigação por sulcos de infiltração e as parcelas foram manejadas de acordo com as recomendações técnicas da cultura, na região. $\mathrm{O}$ ponto de colheita foi determinado com base no secamento das gavinhas adjacentes aos pedúnculos dos frutos.

Foram avaliados o número de dias para o aparecimento da primeira flor masculina (NDM), o número de dias para o aparecimento da primeira flor feminina (NDF), número de gemas da base da planta até a primeira flor masculina (NGM), número da gemas da base da planta até a primeira flor feminina (NGF), comprimento da rama principal (CRP), número médio de frutos por planta (NFP), peso médio de fruto (PMF), teor de sólidos solúveis (TSS), diâmetro transversal do fruto (DTF), diâmetro lon- gitudinal do fruto (DLF) e espessura média da casca do fruto (EMC). Foram avaliadas as cinco plantas centrais e cinco frutos representativos de cada parcela para obtenção das médias das características mencionadas. Os dados foram submetidos à análise de variância e as médias foram comparadas pelo teste de Scott \& Knott (1974) a 5\% de probabilidade.

As medidas de dissimilaridade foram calculadas por meio da distância 
generalizada de Mahalanobis e o agrupamento das linhagens foi realizado utilizando-se o método de Tocher. Com base no método hierárquico de Ward, estabeleceu-se dendrograma ilustrativo da similaridade entre as linhagens. O gráfico de dispersão foi construído a partir dos escores das duas primeiras variáveis canônicas para os 31 genótipos. A contribuição relativa das variáveis para a divergência genética foi determinada utilizando o critério proposto por Singh (CRUZ; REGAZZI, 1997). A obtenção das médias de cada parcela foi realizada utilizando-se o aplicativo Microsoft Excel e os cálculos da análise de variância, teste de comparação de média e divergência genética foram realizados por meio do aplicativo GENES (CRUZ, 2001).

\section{RESULTADOS E DISCUSSÃO}

Houve diferenças significativas entre as linhagens, pelo teste $\mathrm{F}(\mathrm{P}<0,01)$, para todas as características avaliadas, exceto para número de dias para o aparecimento da primeira flor masculina (Tabela 1). As características que mais contribuíram para a divergência entre as linhagens foram número de frutos por planta $(28,8 \%)$, diâmetro longitudinal $(26,4 \%)$, teor de sólidos solúveis $(12,4 \%)$ e peso médio de fruto $(11,9 \%)$, enquanto a que menos contribuiu foi a espessura média de casca $(0,6 \%)$.

Com base nos resultados obtidos pelo cálculo da distância generalizada de Mahalanobis $\left(\mathrm{D}_{\text {ii }}^{2}\right.$ ) (Tabela 2), observouse que as linhagens 87-019.021 e 87019.022 apresentaram a menor magnitude de distância $(1,71)$, sendo portanto as mais semelhantes. Por outro lado, a maior magnitude de distância $(467,94)$ foi observada entre a linhagem 87 019.023 e a cultivar 'Crimson Sweet', sendo este portanto o par mais dissimilar.

O método de agrupamento de Tocher possibilitou o estabelecimento de três grupos. Das oito linhagens oriundas de Pernambuco, sete $(87-019.017 ; 87$ $019.021 ; 87-019.022$; 87-019.023; 87019.027; 97-0251.005 e 97-0251.017) foram incluídas no grupo I, juntamente com a linhagem da Bahia (970249.006), enquanto as 21 linhagens provenientes do Maranhão (91-109.059; 91-109.060; 91-109.087; 91-109.118; 91-

Tabela 2. Medidas mínimas e máximas de dissimilaridade genética entre linhagens de melancia, com base na distância generalizada de Mahalanobis. Petrolina, Embrapa Semi-Árido, 1997.

\begin{tabular}{|c|c|c|c|c|}
\hline \multirow{2}{*}{ Linhagem } & \multicolumn{2}{|c|}{ Mínima } & \multicolumn{2}{|c|}{ Máxima } \\
\hline & $D_{i i}{ }^{2}$ & Linhagem & $D_{i i}{ }^{\prime 2}$ & Linhagem \\
\hline $87-019.017$ & 4,77 & $87-019.023$ & 428,55 & Crimson Sweet \\
\hline $87-019.021$ & 1,71 & $87-019.022$ & 463,10 & Crimson Sweet \\
\hline 87-019.022 & 1,71 & $87-019.021$ & 447,88 & Crimson Sweet \\
\hline $87-019.023$ & 4,77 & $87-019.017$ & 467,94 & Crimson Sweet \\
\hline $87-019.027$ & 6,48 & 87-019.017 & 379,97 & Crimson Sweet \\
\hline $91-109.059$ & 5,13 & $91-079.045$ & 350,65 & Crimson Sweet \\
\hline $91-109.060$ & 5,83 & 91-079.009 & 312,77 & Crimson Sweet \\
\hline $91-109.087$ & 5,04 & $91-109.118$ & 370,28 & Crimson Sweet \\
\hline $91-109.118$ & 5,04 & $91-109.087$ & 345,71 & Crimson Sweet \\
\hline $91-074.015$ & 6,01 & 91-074.012 & 443,94 & Crimson Sweet \\
\hline $91-074.029$ & 15,15 & $91-109.060$ & 273,19 & Crimson Sweet \\
\hline $91-074.012$ & 6,01 & $91-074.015$ & 421,23 & Crimson Sweet \\
\hline $91-079.001$ & 8,54 & 91-079.045 & 338,44 & Crimson Sweet \\
\hline $91-079.008$ & 2,92 & $91-079.060$ & 262,50 & Crimson Sweet \\
\hline $91-079.009$ & 4,55 & $91-079.060$ & 255,77 & Crimson Sweet \\
\hline $91-079.010$ & 11,75 & $91-109.118$ & 320,22 & Crimson Sweet \\
\hline $91-079.044$ & 12,63 & 91-079.009 & 313,14 & Crimson Sweet \\
\hline $91-079.045$ & 5,13 & $91-109.059$ & 330,62 & Crimson Sweet \\
\hline $91-079.060$ & 2,92 & 91-079.008 & 263,85 & Crimson Sweet \\
\hline $91-099.011$ & 7,89 & 91-099.036 & 309,43 & Crimson Sweet \\
\hline $91-099.023$ & 3,84 & $97-0257.005$ & 236,08 & Crimson Sweet \\
\hline $91-099.036$ & 7,89 & $91-099.011$ & 290,33 & Crimson Sweet \\
\hline $97-0247.008$ & 26,33 & $97-0265.021$ & 269,13 & $87-019.023$ \\
\hline $97-0249.006$ & 24,24 & $87-019.027$ & 419,34 & Crimson Sweet \\
\hline $97-0251.005$ & 16,46 & $97-0251.017$ & 217,42 & Crimson Sweet \\
\hline $97-0251.017$ & 12,52 & $87-019.027$ & 298,77 & Crimson Sweet \\
\hline $97-0257.005$ & 3,84 & $91-099.023$ & 223,27 & Crimson Sweet \\
\hline $97-0265.021$ & 18,53 & $97-0257.005$ & 187,86 & $87-019.023$ \\
\hline 97-0263.029 & 19,22 & $91-079.060$ & 350,80 & Crimson Sweet \\
\hline $97-0207.002$ & 6,02 & 91-079.008 & 309,17 & Crimson Sweet \\
\hline Crimson Sweet & 77,44 & $97-0247.008$ & 467,94 & $87-019.023$ \\
\hline
\end{tabular}

074.015; 91-074.029; 91-074.012; 91079.001; 91-079.008; 91-079.009; 91$079.010 ; 91-079.044 ; 91-079.045 ; 91-$ 079.060; 91-099.011; 91-099.023; 91099.036; 97-0257.005; 97-0265.021; $97-$ 0263.029 e 97-0207.002) foram incluídas no grupo II. A linhagem 97-0247.008 e a cultivar Crimson Sweet compuseram o grupo III. A aplicação do método de Ward (Figura 1) resultou na formação de três grupos, sendo que o primeiro grupo coincide exatamente com aquele formado pela técnica de Tocher e os demais diferem apenas pela plotagem da linhagem 97.0265.021 no grupo III, juntamente com a linhagem 97-0247.008 e a cultivar Crimson Sweet.

As estimativas dos autovalores correspondentes às duas primeiras variáveis canônicas explicaram $84,6 \%$ da variação total, permitindo uma descrição satisfatória da divergência genética entre as linhagens por meio da dispersão gráfica dos escores em relação às duas primeiras variáveis canônicas (Figura 2). A análise da dispersão gráfica baseada nas duas primeiras variáveis canônicas permite a formação de quatro grupos bastante semelhantes àqueles obtidos pelas técnicas de agrupamento. Observou-se que os genótipos mais divergentes são as linhagens 87-019.23 e 'Crimson Sweet', sendo que esta última apresentou-se bastante isolada em relação aos demais genótipos. Estes resultados estão de acordo com aqueles obtidos pela distância generalizada de Mahalanobis $\left(\mathrm{D}^{2}{ }_{\mathrm{ii}}\right)$. 


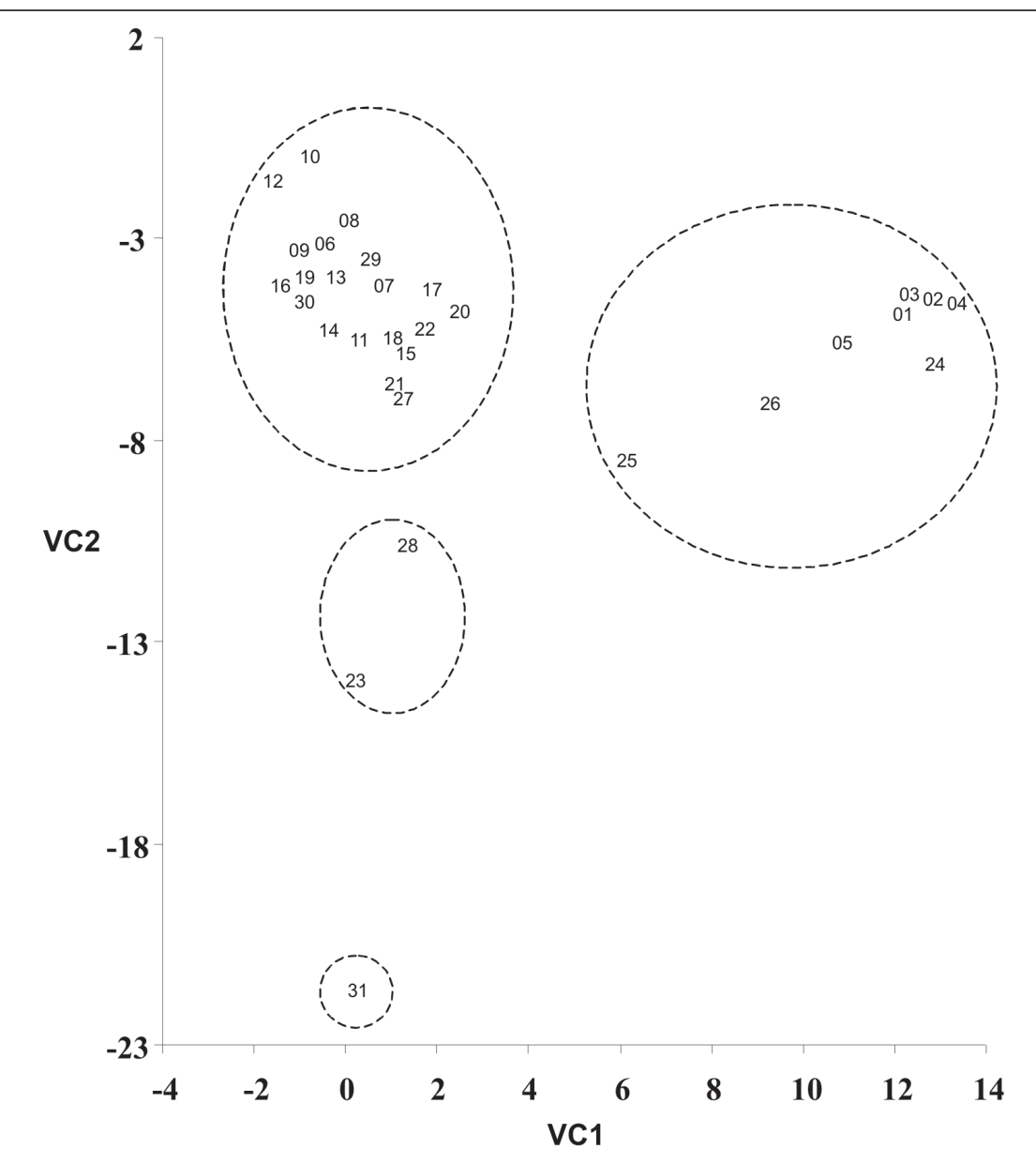

Legenda: $01=87-019.017 ; 02=87-019.021 ; 03=87-019.022 ; 04=87-019.023 ; 05=$ $87-019.027 ; 06=91-109.059 ; 07=91-109.060 ; 08=91-109.087 ; 09=91-109.118 ; 10$ $=91-074.015 ; 11=91-074.029 ; 12=91-074.012 ; 13=91-079.001 ; 14=91-079.008$; $15=91-079.009 ; 16=91-079.010 ; 17=91-079.044 ; 18=91-079.045 ; 19=91-079.060$; $20=91-099.011 ; 21=91-099.023 ; 22=91-099.036 ; 23=97-0247.008 ; 24=97$ $0249.006 ; 25=97-0251.005 ; 26=97-0251.017 ; 27=97-0257.005 ; 28=97-0265.021$; $29=97-0263.029 ; 30=97-0207.002$ e $31=$ Crimson Sweet.

Figura 2. Dispersão gráfica de 31 linhagens de melancia, com base nas duas primeiras variáveis canônicas. Petrolina, Embrapa Semi-Árido, 1997.

Observa-se que os grupos formados diferem pelo nível de melhoramento genético dos genótipos. As linhagens do grupo I foram obtidas a partir de acessos coletados em populações espontâneas, submetidas à seleção natural. No grupo II e III, estão as linhagens oriundas de acessos coletados em pequenas propriedades rurais e em feiras livres, sendo, portanto materiais que têm sofrido além da seleção natural, a seleção massal praticada pelos agricultores. No grupo IV, encontra-se a cultivar Crimson Sweet que deriva de um eficiente programa de melhoramento genético conduzido nos Estados Unidos desde meados do século passado.
A escolha dos progenitores para programas de hibridação que visem à ampliação da variabilidade da espécie para o melhoramento genético por meio de seleção nas gerações segregantes deve levar em consideração a divergência genética, o desempenho per se dos progenitores e a complementaridade alélica. Desse modo, deduz-se que os cruzamentos mais promissores para a formação de populações base, com ampla variabilidade e compostas de genótipos superiores, serão aqueles realizados entre a cultivar Crimson Sweet e as linhagens do grupo II, que além de bastante divergentes, apresentaram bom desempenho com relação às características avaliadas, sobretudo quanto ao peso de frutos e ao teor de sólidos solúveis (Tabela 1). Por outro lado, cruzamentos envolvendo a cultivar Crimson Sweet e as linhagens do grupo I, serão interessantes para a obtenção de populações de plantas prolíficas e de frutos pequenos, haja vista que as linhagens deste grupo apresentaram maior número de frutos por planta e menor peso de fruto (Tabela 1). É esperado que o cruzamento entre 'Crimson Sweet' e a linhagem 97.0247.008 resulte em menor variabilidade se comparado aos cruzamentos anteriores.

\section{LITERATURA CITADA}

ASSIS, J.G.A. Estudos Genéticos no Gênero Citrullus. 1994. 98 F. (Tese mestrado) - UNESP, Jaboticabal.

CRUZ, C.D., REGAZZI, A.J. Modelos Biométricos Aplicados ao Melhoramento Genético. Viçosa: UFV, Imprensa Universitária, 1997. 390 p.

CRUZ, C.D. Programa Genes: aplicativo computacional em genética e estatística. Viçosa, MG: UFV, 2001. 648 p.

CRUZ, C.D., VENCONVSKY, R. Comparação de alguns métodos de análise dialélica. Revista Brasileira de Genética. Ribeirão Preto, v.12, n.2, p.425-436, 1989.

FAO. FaoStat Database Results. Disponível em: <http://www.fao.org.>. Acessoo em 18 jun. 2003. GERALDI, I.O.; MIRANDA-FILHO, J.B. Adapted models for the analysis of combining ability of varieties in partial diallel crosses. Revista Brasileira de Genética. Ribeirão Preto, v.11, p.419-430, 1988.

GUERRA-SANZ J.M. Citrullus simple sequence repeats markers from sequence databases. Molecular Ecology Notes, n.2, p.223-225. 2002. IBGE. Produção Agrícola. Disponível em: <http:/ /www.sidra.ibge.gov.br/cginbin>. Acesso em 20 mai. 2003.

JARRET, R.L.; MERRICK, L.C.; HOLMS, T.; EVANS, J. Simple sequence repeats in watermelon [Citrullus lanatus (Thunb.) Matsum \& Nakai]. Genome, v.40, p.433-449. 1997.

LEE, S.J.; PARK, K.W.; HONG Y.P. Detection of genetic diversity using RAPD-PCR and sugar analysis in watermelon [Citrullus lanatus (Thunb.) Mansf.] germplasm. Theoretical and applied genetics, v.92, p.719-725, 1996.

QUEIRÓZ, M.A.; SOUZA, F.F.; Melhoramento de melancia para diferentes padrões de fruto e teor de açúcar. In: CONGRESSO BRASILEIRO DE OLERICULTURA, 38º, 1998, Petrolina-PE. Resumos... Petrolina, PE: EMBRAPA-CPATSA/ SOB, 1998. Não paginado.

QUEIRÓZ, M.A.; DIAS, R.C.S.; SOUZA, F.F.; FERREIRA, M.A.J.F; ASSIS, J.G.A.; BORGES, R.M.E.; ROMÃO, R.L.; RAMOS, S.R.R.; COSTA, M.S.V.; MOURA, M.C.C.L. Recursos genéticos e melhoramento de melancia no Nordeste brasileiro. In.: QUEIRÓZ, M.A.; GOEDERT, C.O.; RAMOS, S.R.R., ed. Recursos Genéticos e Melhoramento de Plantas para o Nordeste brasileiro. (on line). Petrolina: Embrapa Semi-Árido/ Brasília-DF: Embrapa Recursos Genéticos e Biotecnologia, 1999. Disponível em: <http:// www.cpatsa.embrapa.br>.

SCOTT, A.J.; KNOTT, M. A cluster analysis method for grouping means in the analysis of variance. Biometrics, v.30, p.507-512, 1974. 